\title{
Transcriptional activation of mouse retrotransposons in vivo: specific expression in steroidogenic cells in response to trophic hormones
}

\author{
Rachel Schiff, Ahuva Itin, and Eli Keshet \\ Department of Virology, The Hebrew University, Hadassah Medical School, Jerusalem 91010 Israel
}

\begin{abstract}
Transcription of cellular retrotransposons is induced by a variety of physiological stimuli. We have used in situ hybridization analysis to determine the cell types in which mouse retrotransposons are transcriptionally activated in vivo under physiological conditions. Here, we report that VL30 retrotransposons are specifically expressed in steroidogenic cells within all four endocrine tissues engaged in synthesis of steroid hormones in response to the respective pituitary-derived trophic hormones. These tissues include ovarian steroidogenic theca cells and lutein cells of the corpus luteum, testosterone-producing Leydig cells of the testis, steroidogenic cells confined to the zona reticularis of the adrenal cortex, and progesterone-producing cells of the placenta. In the course of preovulatory follicular development and maturation, the profile of cells expressing the retrotransposon shifted in parallel to the changing profiles of the leutinizing hormone (LH)-induced steroidogenic output of the respective cells. Expression of VL30 in both male and female gonads was shown to be greatly stimulated by external administration of gonadotropins. In vitro studies using a LH-responsive Leydig cell line have confirmed that expression of the resident retrotransposons is gonadotropin dependent. Run-off transcription assays have indicated that activation is at the transcriptional level. To allow molecular access to gonadatropin-activated transcription units, the long terminal repeat (LTR) regulatory domains were cloned from VL30 cDNAs of LH-induced ovaries. Through the use of reporter gene constructs and transfection experiments it was shown that expression of these elements in steroidogenic cells is LH dependent. Furthermore, cAMP, a known mediator of trophic hormone responses, could replace the hormone for inducibility. Transfection studies have also shown that the retrotransposon LTRs may function as hormone-activated enhancers conferring a LH-dependent phenotype on a surrogate transcription unit. These studies have thus demonstrated that the transcriptional activation of resident retrotransposons in vivo is a dynamic process that can be modulated by gonadotropins and have the potential of imposing this phenotype on adjacent cellular genes.
\end{abstract}

[Key Words: Retrotransposons; VL30; in situ hybridization; steroidogenesis; gonadotropins]

Received August 31, 1990; revised version accepted January 15, 1991.

The mammalian genome harbors a large number of endogenous proviruses that are genetically related to infectious retroviruses. In addition, mammalian chromosomes contain other types of genetic elements, termed retrotransposons, that are genetically unrelated, or only distantly related, to known infectious retroviruses but possess, nevertheless, structural hallmarks of proviruses. Notably, retrotransposons are distinguished by long terminal repeats (LTRs) housing the transcriptional regulatory domains of the element. In mice, where these provirus-like elements were best studied, at least six distinct retrotransposon families, each containing from 100 to several thousand members, were discovered (for review, see Keshet et al. 1990). Transcriptional activation of retrotransposons is a prerequisite and often a ratelimiting step in retrotransposition (Boeke et al. 1985).
The tissue- and cell-type specificities of retrotransposon LTRs therefore determine the nature of cells in which retrotranspositions or retrotransposon-mediated recombinations may take place. Retrotransposons may also affect the expression of nearby cellular genes, from their sites of residence through the activity of their transcriptional enhancers. Hence, cell-type-specific or hormoneresponsive retrotransposon enhancers may determine the pattern of expression of adjacent cellular genes. For example, it has been shown recently that certain mouse retrotransposons may impose hormone responsiveness upon an adjacent cellular gene (Stavenhagen and Robins 1988).

The current study focuses on a particular murine retrotransposon family designated the VL30 elements (Howk et al. 1978; Besmer et al. 1979; Keshet et al. 1980). 
Over 100 copies of these potentially transmissible elements are scattered throughout the mouse genome. The evolutionary plasticity of retrotransposon LTRs, in general, and of VL30 LTRs, in particular, has led to a situation where different members of the family often possess structurally diverse LTRs that display different transcriptional specificities (Itin and Keshet 1986a). A characteristic feature of some retrotransposon families is the remarkable inducibility of their transcription by a wide range of agents and physiologic stimuli. For example, VL30 retrotransposons are activated under a variety of experimental conditions. This is reflected in the repeated incidental selection of VL30 cDNA clones in substructive screening procedures aimed at selecting inducible genes. Thus, VL30 elements were selected as genes in which expression is turned on after epidermal growth factor (EGF) stimulation of quiescent mouse embryo cells in culture (Foster et al. 1982); VL30 cDNAs were selected as growth-specific elements expressed in SV40transformed, nonconfluent cells but not in the confluent, contact-inhibited parental cells (Singh et al. 1985); VL30 cDNAs were picked up in a selective procedure aimed at identifying genes in lymphoid cells activated by glucocorticoids (Harrigan et al. 1989); VL30 elements were shown to be activated by chemical carcinogens (Hsieh et al. 1987), whereas other studies have demonstrated a dramatic (500-fold) induction of VL30 expression under conditions of anoxia (Anderson et al. 1989).

Whereas the patterns of retrotransposon expression have been widely studied in cell culture systems, the activation of mammalian retrotransposons in vivo under conditions that allow the correct systemic affects and preserve the authentic cellular interactions has been only poorly studied. Reasoning that in situ hybridization analysis might point at distinct physiologic settings in which resident retrotransposons are transcriptionally activated, we embarked on a comprehensive in situ hybridization analysis, using specimens derived from mouse organs and embryos. Here, we report our observations that VL30 retrotransposons are activated in vivo specifically in steroidogenic cells within the male and female gonads, the adrenal cortex, and the placenta, that is, in all four endocrine tissues that specialize in synthesis and secretion of steroid hormones. Cyclic changes in both levels of expression and types of expressing cells were observed within the female reproductive system in correspondence to cyclic changes in gonadotropin levels. Our studies further suggested that transcriptional activation of resident VL30s in different steroidogenic organs is mediated by the action of the respective trophic hormone. Transfection experiments with selected cloned LTR units indicated that inducibility is an inherent property of the LTRs. Further experiments suggested that these LTRs may potentially impose gonadotropin inducibility on adjacent cellular genes.

\section{Results}

To elucidate the patterns of VL30 expression we carried out a comprehensive in situ hybridization analysis. This methodology has the advantage that expression confined to individual cells within a tissue can be detected by a screening procedure that covers a large number of tissues and embryonic organs. Moreover, the effects of physiologic treatments (e.g., the systemic administration of hormones) on transcription can be monitored without disturbing the natural cellular interactions.

As a source of a VL30-specific sequences we used a 350-bp-long LTR fragment derived from a previously characterized genomic clone. We have shown previously that certain VL30 clones are, in fact, mosaic elements composed of more than one retrotransposon type (Itin et al. 1983). The use of a defined LTR sequence as a probe eliminated the possibility that the probe employed included sequences that could cross-hybridize with other genomic elements. VL30 cRNA probes labeled with ${ }^{35} \mathrm{~S}$ in either antisense or sense orientations were synthesized. Embryos and organs were fixed in 4\% paraformaldehyde, embedded, frozen, and sectioned. Frozen sections $(10 \mu \mathrm{m})$ were processed for in situ hybridization and were hybridized under stringent conditions, as described in Materials and methods.

To include as many cell types as possible in each section examined, we initiated our "shotgun" screening by examining serial sections obtained from whole newborn mice or from late embryonic stages $[>17$ days postcoitum (p.c.)]. This was followed by focusing on tissues or organs that gave the most intense autoradiographic signals.

\section{Expression of VL30 in the adrenal cortex}

Figure 1A shows an example of a parasagittal section through a 17.5-day-old mouse embryo. Expression of VL30s could be detected in several tissues, primarily in the epithelial tissue layers of most internal organs (not evident in the low magnification view provided). However, it was clearly seen that the predominant site of expression, with highest detectable levels of VL30 RNA, is the embryonic adrenal gland. Histologic details of an embryonic adrenal gland are better seen in the higher magnification view (Fig. IB). VL30 RNA was mostly detectable in the provisional cortex residing underneath the outermost cortical layers (layers that at later developmental stages will give rise to the permanent cortex). The major function of the provisional cortex is the production and secretion of the sulfate conjugates of androgens (which are converted to active androgens and estrogens in the placenta). Because the provisional cortex is destined to undergo involution after birth and to be replaced by the permanent cortex, we wished to determine the sites of VL30 expression in the mature adrenal gland. Adrenal glands from adult mice were hybridized in situ with the VL30-specific probe. As can be seen in Figure $1 \mathrm{C}$, expression of VL30 in the adrenal gland was restricted to the inner zone of the adrenal cortex, the zona reticularis. No hybridization signals were detected in the adrenal medula. Strikingly, no hybridization signals were detected in the two outer cortical zones, the zona glomerulosa and the zona fasciculata, despite the fact 

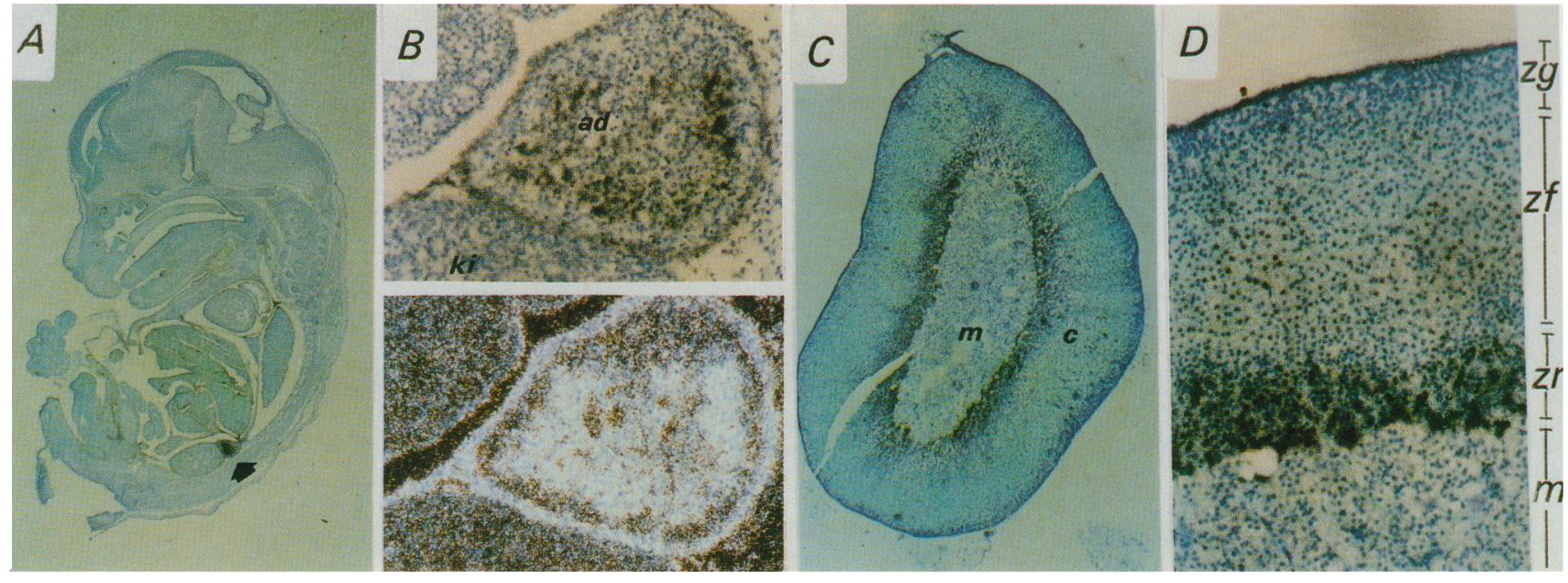

Figure 1. Expression of VL30 in the embryonic and adult adrenal gland. (A) A parasagittal section through a 17.5-day-old mouse embryo. The arrow indicates the adrenal gland (magnification, 4.6×). $(B)$ Enlargement of the adrenal gland shown in $A$ (magnification, $74 \times)$. (Top and bottom) Bright-field and dark-field images, respectively. (C) A section through the adrenal gland of an adult mouse (magnification, 24.6×). (D) Enlargement of a cortical region from the gland shown in $C$ (magnification, $99 \times$ ). Sections were processed and hybridized in situ with a VL30-specific probe, as described in Materials and methods. Autoradiographic exposure was for 5 days. Sections were counterstained with toluidine blue. (ki) Kidney; $(\mathrm{ad})$ adrenal; $(\mathrm{c})$ cortex; $(\mathrm{m})$ medula; $(\mathrm{zg})$ zona glomerulosa; $(\mathrm{zf})$ zona fasciculata; $(z r)$ zona reticularis.

that all three cortical zones are stimulated by adrenocorticotropic hormone (ACTH) to produce steroid hormones. We assume that the highly restricted pattern of VL30 expression in the adrenal cortex reflects subspecialization of cells within the three cortical zones with respect to their biochemical synthetic capacities. Specifically, the zona glomerulosa is known to synthesize mineralocorticoids and the zona fasciculata is primarily engaged in synthesis of glucocorticoids, whereas cells of the zona reticularis are known to produce, among other steroids, 17 ketosteroids (Simpson and Waterman 1988). However, because the precise pattern of distribution of the different hormone-producing cell types within the adrenal cortex is not known, it is difficult to establish functional correlates.

\section{Expression of VL30 in embryonic implantation sites} and in the placenta

Analysis of VL30 expression during the early postimplantation period (6.5-10.5 days p.c.) was carried out using longitudinal sections through the entire uterus in the pregnant mouse. This procedure enabled us to compare VL30 RNA levels in all tissues constituting the implantation sites, including those of the embryo proper, extraembryonic tissues, and maternal tissues. Figure 2, A and $B$, shows sections through implantation sites of early conceptuses shortly after implantation (6.5 days p.c.). VL30-expressing cells were asymmetrically distributed with highest levels of RNA detected in the mural side of the embryo. The higher magnification view of VL30-expressing cells (Fig. 2C) shows that expression is localized mostly in giant trophoblast cells surrounding the embryo or embedded in the decidua, as well as in maternal decidual cells. These results are consistent with observations that certain steroidogenic enzymatic activities appear shortly after implantation and that they are found primarily in association with mural trophoblast giant cells (Soares et al. 1985). This pattern of distribution also grossly parallels the distribution pattern of cells that abundantly transcribe mRNA of the key steroidogenic enzyme cholesterol side-chain cleavage cytochrome p450 (p450 scc $_{\text {) }}$ (R. Schiff et al., in situ hybridization data not shown). At later gestational stages, the predominant site of VL30 transcription shifted to trophoblast cells of the chorioallantoic placenta (Fig. 2D). The latter is known to be the major site of placental progesterone synthesis. These results suggest that within implantation sites and later in the placenta, VL30-expressing cells are predominantly steroidogenic cells.

\section{Expression of VL30 in the gonads}

The nature of the cells that we have identified as VL30expressing cells in the adrenal gland and the placenta hinted that VL30 elements might be activated in additional types of steroidogenic cells. Therefore, we examined the two other major steroidogenic organs: the male and female gonads. Ovaries derived from mature, randomly cycling female mice were sectioned and hybridized with the VL30-specific probe. Hybridization signals were detected predominantly in theca cells and in the corpus luteum. An example of theca cells expressing VL30 RNA is shown in Figure 3A. Theca cells are seen arranged in several concentric layers at the periphery of ovarian follicles and are clearly distinguishable from granulosa cells that occupy a more central follicular position. It is clearly seen that in the particular follicle 


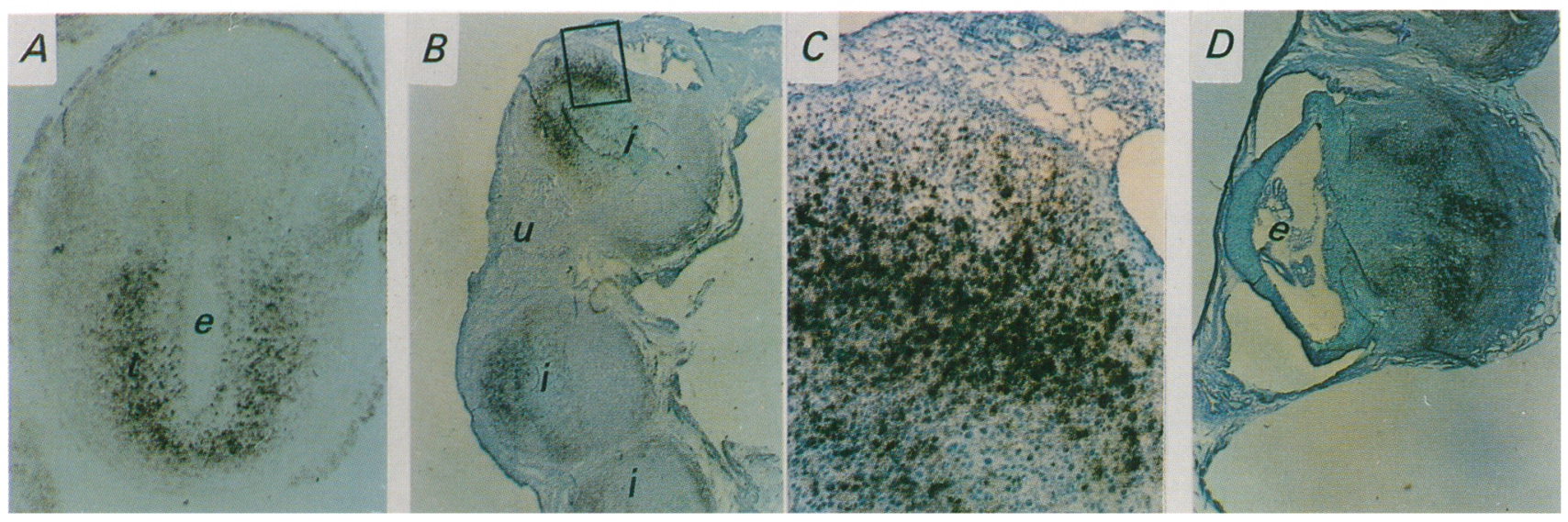

Figure 2. Expression of VL30 in embryonic implantation sites and placenta. (A) A section through an implantation site of a 6.5day-old embryo (magnification, $15.4 \times$ ). (B) A section through several adjacent implantation sites (6.5 days p.c.) also showing interimplantation uterine regions (magnification, $7.7 \times) .(C)$ Enlargement of the boxed area shown in $B$ (magnification, $57.8 \times) .(D)$ A section through an implantation site at 9.5 days p.c. (magnification, $9.2 \times$ ). In situ hybridization with a VL30-specific probe as described in Fig. 1. (e) Embryo; (i) implantation site; (u) interimplantation uterine region.

shown in Figure 3A, VL30 expression is restricted to theca cells. Neither granulosa cells nor the oocyte were labeled. A second site of VL30 expression detected in ovaries derived from randomly cycling mice was the corpus luteum (Fig. 3B). The corpus luteum is formed after ovulation in the cortical region of the ovary from granulosa cells and remaining theca cells. In the nonpregnant mouse, the corpus luteum functions during the luteal stage of the estrous cycle as a temporary endocrine gland. It is noteworthy that both theca cells and lutein cells of the corpus luteum are steroidogenic cells producing predominantly testosterone and progesterone, respectively, in response to the stimulating action of luteinizing hormone $(\mathrm{LH})$ produced in the pituitary gland.

\section{Expression of VL30 in the ovary is hormone inducible}

The detection of VL30 RNA specifically in ovarian cells that produce steroids in response to LH suggested that the regulatory elements of these retrotransposons respond to the stimulatory action of the gonadotropin hormone. It is well established that follicular development and maturation is controlled by the pituitary gonadotro- pins, follicle-stimulating hormone (FSH) and LH. Several cell types in the ovary, including the theca interna, granulosa, and interstitial cells located between follicles, are known to participate in the synthesis of steroids. All three cell types are regulated by the gonadotropins in a complex manner, resulting in a pattern that changes concomitantly with the progression of follicles toward ovulation (for review, see Richards 1980).

To determine whether VL30 expression in each of these cell types is correlated with follicular growth and maturation, we analyzed ovaries that had been synchronized for follicular growth by the external administration of gonadotropins. The ovaries were hyperstimulated by injecting a large dose of pregnant mare's serum gonadotropin (PMSG), followed by injection of human chorionic gonadotropin (hCG) $44 \mathrm{hr}$ later (hCG mimics the endogenous LH surge). In this procedure a relatively large cohort of follicles ensues growth in a synchronous manner. Because pronounced changes in the steroidogenic activity of different ovarian cell types is known to occur after the LH surge, we focused our analyses on these stages. We first compared relative levels of VL30 RNA in ovaries at successive stages of the cycle by per-
Figure 3. Expression of VL30 in steroidogenic cells of the ovary. In situ hybridizations were carried out on sections derived from randomly cycling female mice (10 weeks of age). (A) A section through an ovarian follicle (magnification, 120 $\times$ ). (B) A section through a corpus luteum (magnification, 90×). (oo) Oocyte; (g) granulosa cells; $(t)$ theca cells; (cl) corpus luteum.
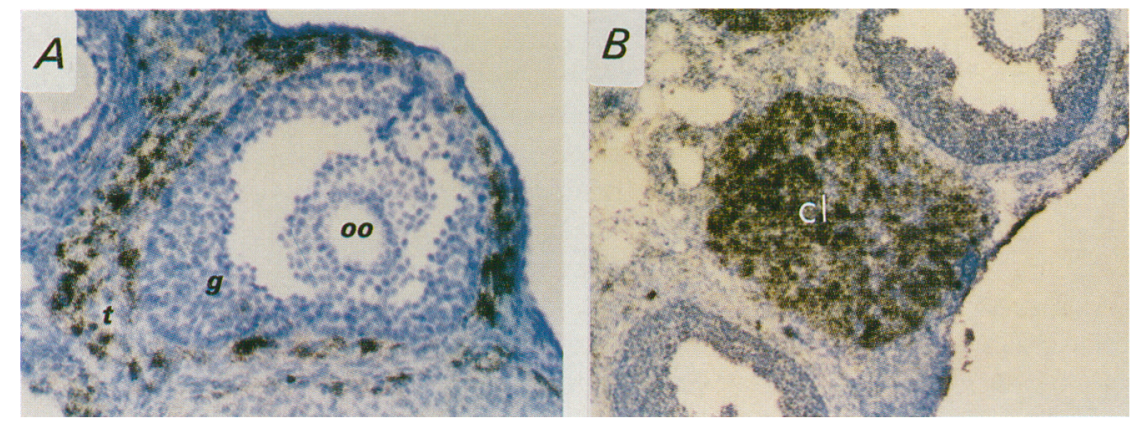
forming an RNA blot analysis (Fig. 4). Ovaries of AKR/J mice were withdrawn at different times after administration of $\mathrm{hCG}$, and RNAs were extracted. AKR/J mice were chosen to allow comparison with the expression profile of activatible endogenous ecotropic proviruses that reside in the DNA of this strain (Jenkins et al. 1982). Ecotropic proviruses were shown previously to display a theca cell-specific expression in other mouse strains (Panthier and Condamine 1987; Lock et al. 1988; Panthier et al. 1989), an observation extended by us to AKR/J mice (data not shown). hCG caused a dramatic elevation in VL30 expression, with maximal levels detected between 5 and $10 \mathrm{hr}$ after hCG administration and a sharp decline observed at postovulatory stages [ovulation in mice takes place 10-12 hr post-hCG (Hogan et al. 1986)). When the same RNA preparations were hybridized with the ecotropic virus-specific probe (see Materials and methods) the expected $35 \mathrm{~S}$ and subgenomic 21S RNA species were detected, but RNA levels remained constant throughout the cycle (Fig. 4B). This result indicated that unlike the ecotropic provirus, expression of VL30 retrotransposons in the ovary is responsive to gonadotropin stimulation.

To follow the kinetics of preovulatory VL30 expression separately in each steroidogenic cell type, ovaries were withdrawn at different times after hCG administration, fixed, sectioned, and hybridized in situ with the VL30-specific probe. As can be seen in Figure 5A, the in situ hybridization pattern was in complete agreement with the RNA blot analysis results (Fig. 4), with respect to the timing of maximal VL30 expression (observed at 5-10 hr after hCG administration). Examinations of each of the expressing cell types have indicated that concomitantly with the progression of follicles toward ovula-

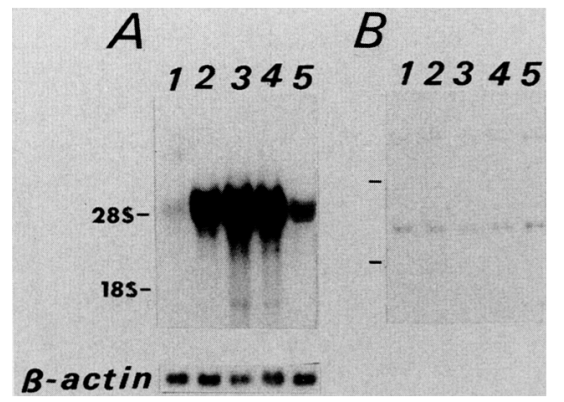

Figure 4. Expression of VL30 retrotransposons and ecotropic proviruses in synchronized hyperstimulated ovaries. Hyperstimulation of ovaries was achieved by injecting AKR/J mice with gonadotropins, as described in Materials and methods. Ovaries were removed for RNA extraction at the following times: (Lane 1) $44 \mathrm{hr}$ after PMSG injection $10 \mathrm{hr}$ for hCG injection); (lane 2) $2.5 \mathrm{hr}$ after hCG injection; (lane 3) $5 \mathrm{hr}$ after hCG injection; (lane 4) $10 \mathrm{hr}$ after hCG injection; (lane 5) $24 \mathrm{hr}$ after hCG injection. For each RNA preparation a pool of ovaries, obtained from five animals, was used. Procedures of RNA blot hybridization were as described in Materials and methods. Filters were rehybridized with a $\beta$-actin-specific probe for standardization. rRNAs served as size markers. (A) VL30-specific probe; $(B)$ ecotropic virus-specific probe. tion, additional cell types (other than theca cells) expressed VL30 RNA. These types included interstitial cells, granulosa cells and, just preceding ovulation, the cumulus cell (Fig. 5B,C). Interestingly, the wavelike pattern of cells acquiring the capacity to express VL30 RNA grossly paralleled the acquisition of steroidogenic activity by these cells. This was manifested by the wavelike activation of the steroidogenic enzyme $\mathrm{p} 450_{\mathrm{scc}}$ (Orly 1989). Thus, both temporal and spatial specificities of VL30 expression during the estrous cycle suggest a correlation between maximal inducibility of the retrotransposon and maximal gonadotropin-induced steroidogenic activity of the cell.

\section{VL30s are transcriptionally activated in testicular Leydig cells in response to $\mathrm{LH}$}

We wished to determine whether stimulation of VL30 expression by gonadotropins in the female gonad also could be demonstrated in the male gonad. In the testis, the targets of $\mathrm{LH}$ action are specialized interstitial cells, termed Leydig cells, that elaborate the male steroid hormone testosterone. In situ hybridization of sectioned testes derived from untreated AKR/J mice revealed a barely detectable hybridization signal over Leydig cells /a low hybridization signal was detected over spermatids within the seminiferous tubules) (Fig. 6A,B). A dramatic increase in VL30 expression was observed in sections derived from the testes $5 \mathrm{hr}$ after injection of hCG. VL30 RNA accumulated specifically in Leydig cells. Leydig cells are seen scattered in the interstices among the seminiferous tubules, along with other components of the connective tissue (Fig. 6C). Again, the gonadotropin had no stimulatory effect on the level of expression of the endogenous ecotropic proviruses (data not shown).

To establish that VL30 elements are induced in Leydig cells by LH, we extended our in vivo analysis to a cell culture system. The MLTC-1 line, derived from a murine Leydig cell tumor, was used. This cell line was employed because it produces progesterone in a dose- and time-dependent manner following addition of $\mathrm{LH}$ and because its gonadotropin-responsive adenylate cyclase system has been well characterized (Rebois 1982). The effect of LH administration on accumulation of VL30 RNA was examined by using RNA blot analysis (Fig. 7A). The gonadotropin-induced expression of resident VL30 elements was in a time-dependent manner, with maximal expression observed $5 \mathrm{hr}$ after administration of LH. The transient nature of VL30 RNA accumulation is consistent with the gradual decline in the hormoneinduced cAMP levels observed in this cell line (Rebois 1982).

To determine whether activation of the retrotransposon is at the transcriptional level, we performed a run-on transcription analysis by using nuclei isolated from control and LH-treated MLTC-1 cells (see Materials and methods). As can be seen in Figure 7B, significantly more VL30 RNA was labeled in LH-treated Leydig cells than in nontreated cells /estimated as fivefold on the basis of 


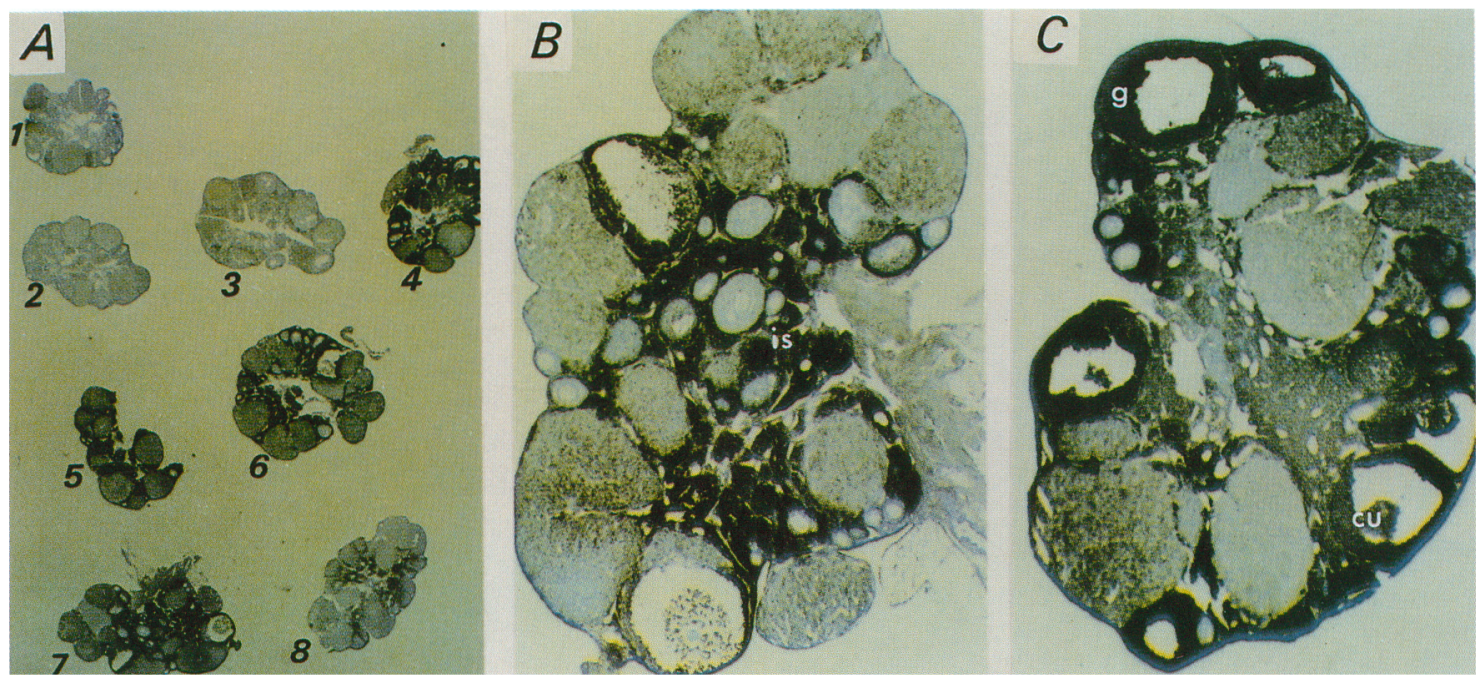

Figure 5. Cell-type specificity of VL30 expression in ovaries at different stages of the estrous cycle. $(A)$ Ovaries were removed to the fixative at the indicated times after hCG injection. The eight ovaries shown were coembedded in O.C.T., cosectioned, and hybridized in situ under the same coverslip. This procedure allowed a visual comparison of the relative levels of VL30 mRNA expressed during successive stages of the estrous cycle. (1 and 2) Ovaries derived from randomly cycling females; (3) $0 \mathrm{hr}$ for hCG (44 hr after PMSG); $(4$ and 5$) 5 \mathrm{hr}$ after hCG; $(6$ and 7$) 10 \mathrm{hr}$ after hCG; $(8) 24 \mathrm{hr}$ after hCG. Magnification $5.2 \times$. (B and $C)$. Higher magnification views $(21 \times)$ of ovarian sections at late preovulatory stages (10 and $12 \mathrm{hr}$ after hCG, respectively). (is) Interstitial cells; (g) granulosa cells; (cu) cumulus cells.

densitometric tracing of the autoradiogram|. Thus, we conclude that, at least in part, activation of VL30 expression is at the transcriptional level.

\section{Isolation and characterization of gonadotropin-responsive VL30 transcription units}

The data reported above clearly demonstrated that resident VL30 elements are transcriptionally activated in response to gonadotropins. However, because VL30 elements occupy $>100$ cellular loci, it remained to be determined whether this phenotype is dictated by regulatory domains of the LTR or, alternatively, whether
LH responsiveness is imposed by cellular sequences flanking a particular VL30 element. The obvious experiments addressing this issue, namely, to reintroduce cloned LTR-reporter gene constructs into steroidogenic cells, have been hampered by the complexity of VL30 LTRs. Sequence analysis of LTRs from randomly selected VL30 genomic and CDNA clones have shown that different elements possess nonidentical LTR units (Itin and Keshet 1986a). Assuming, therefore, that only a subset of VL30 elements are LH inducible, we undertook the task of sorting LH-inducible VL30 elements from nonresponsive ones. To this end we exploited the unique situation that, contrary to bona fide cellular genes, all se-

Figure 6. Activation of VL30 retrotransposons in testicular Leydig cells. $(A$ and $B)$ Testes were removed from a control mouse $(-\mathrm{LH})$ and from a mouse that had been injected with hCG $(+\mathrm{LH})$ (see Materials and methods). Testes were coprocessed, sectioned, and hybridized in situ on the same slide. The same field was photographed under brightfield $(A)$ and dark-field $(B)$ illuminations (magnification, $9.2 \times) .(C)$ High-magnification $(82.8 \times)$ of the gonadotropin-stimulated testis shown in $A$. Note that hybridization signals are predominantly over Leydig cells (ly), and only minor hybridization is detectable over spermatocytes and spermatids (sp).

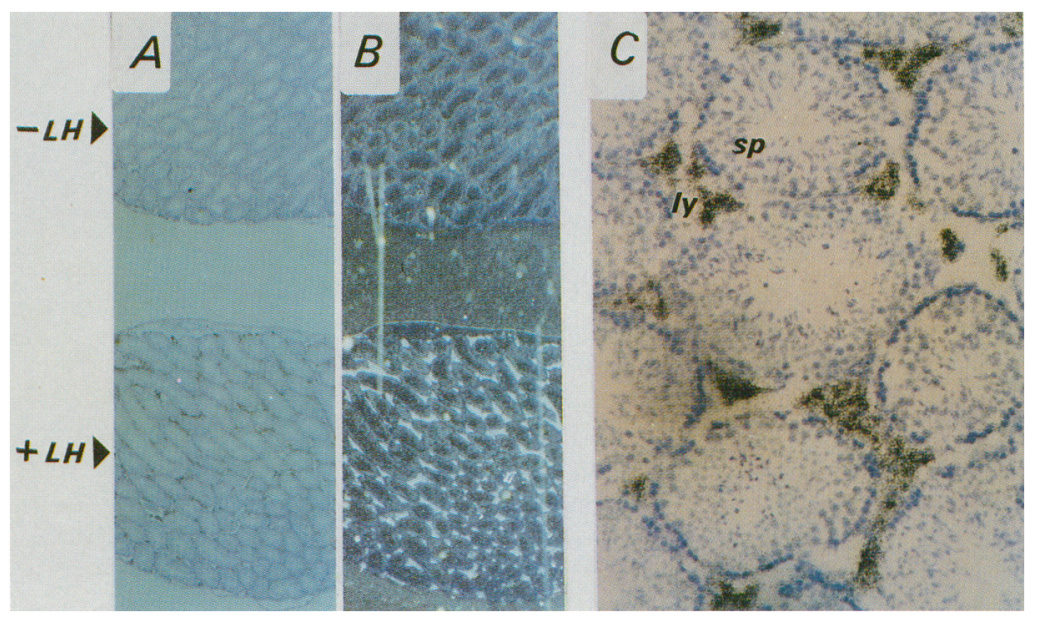




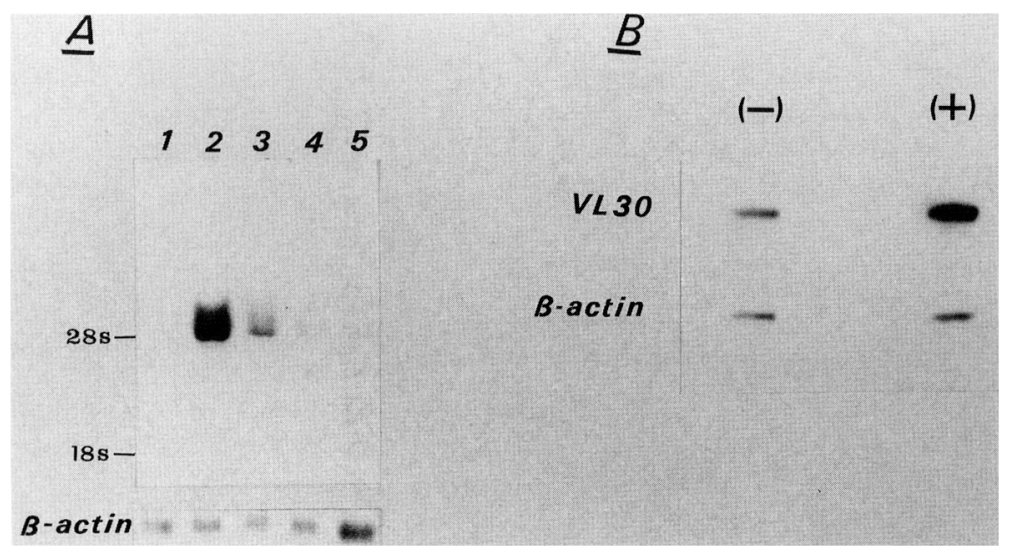

Figure 7. Transcription of resident VL30 retrotransposons in a Leydig cell line is $\mathrm{LH}$ inducible. $(A) \mathrm{LH}$ $(100 \mathrm{ng} / \mathrm{ml})$ was added to the medium of subconfluent cultures of MLTC-1 cells. Cells were harvested at different times after LH addition; RNAs were extracted and analyzed by Northern blot hybridization. (Lane 1) No LH added; (lane 2) $5 \mathrm{hr}$ after LH addition; (lane 3) $10 \mathrm{hr}$ after $\mathrm{LH}$; (lane 4) $10 \mathrm{hr}$ after $\mathrm{LH}$; (lane 5) NIH3T3 cells. (B) Nuclear run-off transcription analysis performed on nuclei isolated from LH-treated lat $5 \mathrm{hr}$ after $\mathrm{LH}$ addition) $(+)$ and untreated (-) MLTC-1 cells. Nuclear RNAs were hybridized with immobilized VL30 DNA or with a control $\beta$-actin DNA. quence domains regulating transcription of retrotransposons are contained in the mRNA. This provided an opportunity to isolate the transcriptional regulatory domains of inducible elements without the need of subjecting a large number of randomly selected genomic clones to a functional analysis.

For cloning LH-induced cDNAs, polyadenylated RNAs were extracted from ovaries withdrawn $5 \mathrm{hr}$ after administration of hCG, reverse-transcribed, and used for construction of a cDNA library in a $\lambda$ gt10 vector (see Materials and methods). We reasoned that because the levels of VL30 RNA at this stage are about two orders of magnitude higher than in the noninduced state (see Fig. 4), most VL30 cDNA clones isolated from this library will contain U3 domains that confer gonadotropin inducibility. Randomly selected VL30 cDNA clones were thus sequenced and used in subsequent functional studies.

Figure 8 shows the nucleotide sequences of the U3 and R LTR regions of two clones, termed VL.ovl and VL.ov2. The two U3 sequences share many structural features with each other, as well as with previously characterized LTRs. The two U3 regions differ in two main points: VL.ovl contains three tandem copies of a basic, 35-bplong repeated unit (the sequence indicated by a solid box), whereas VL.ov2 contains only two tandem copies of the repeat; a 40-bp-long sequence element (the sequence indicated by a dotted box) is inverted in VL.ov2 relative to its orientation in VL.ov1 (this sequence element is itself bounded by an inverted repeat). Thus, excluding a more divergent $5^{\prime}$ segment of $\sim 70 \mathrm{bp}$, the two U3 regions share most sequence domains that may interact with regulatory proteins. Putative TATA and CAT boxes were marked as reference points as they are present in all VL30 LTRs that have been sequenced at the same relative distances from the transcription start site. Additional sequences that resemble consensus binding sites of known trans-acting proteins can be recognized in the U3 domains, but whether they indeed serve as functional binding sites remains to be determined (a study in progress).

To ascertain that the retrotransposons from which the VL.ov1 and VL.ov2 LTRs were derived are LH inducible, we determined whether the LTRs are capable of conferring $\mathrm{LH}$ responsivness on a surrogate reporter gene. Re- porter gene constructs were made in which a promoterless chloramphenicol acetyltransferase (CAT) gene was ligated downstream of the U3-R sequences shown in Figure 8, and DNAs were transfected into MLTC-1 Leydig cells. (for details, see Materials and methods). When

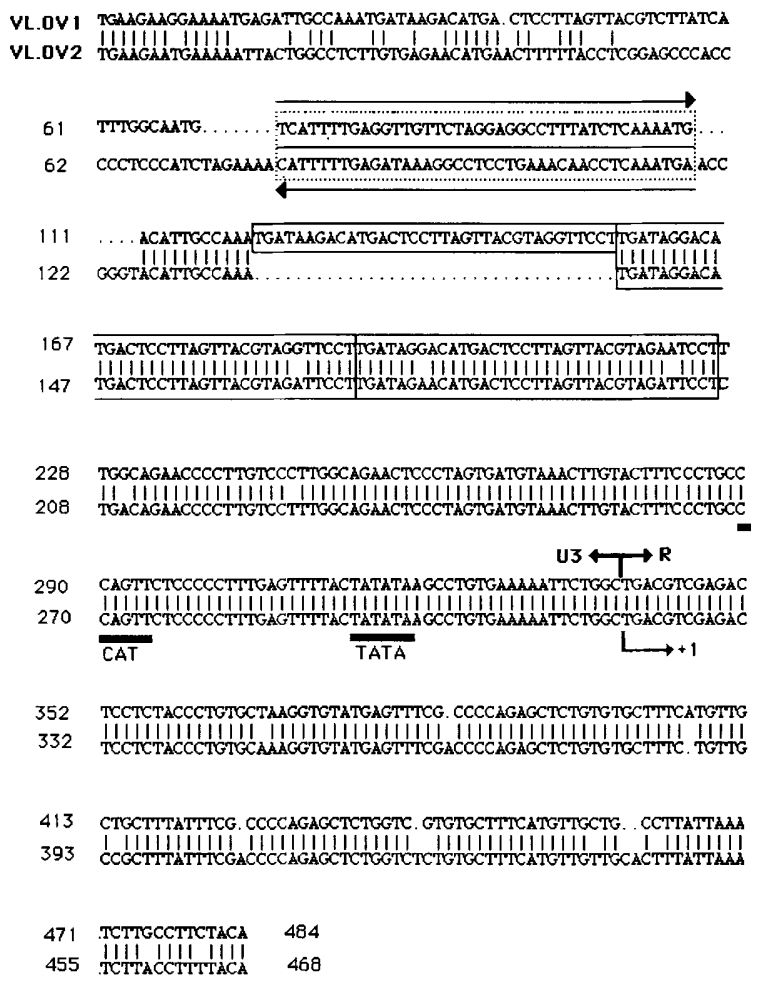

Figure 8. U3-R sequences of VL30 cDNA clones isolated from hCG-stimulated ovaries. Procedures for in vivo stimulation with hCG, construction of a cDNA library from hCG-stimulated ovaries, selection of VL30 clones, and sequencing procedures were carried out as described in Materials and methods. The U3 and R regions of two randomly selected VL30 cDNA clones (VL.ov1 and VL.ov2) are shown. The two sequences were aligned to emphasize sequence homologies. Solid boxes mark a 35-bp-long tandemly repeated sequence; dotted boxes mark a 40-bp-long sequence element present in both cDNAs in inverted orientations. The U3/R junctions and the TATA and CAT boxes are also depicted. 
administered, $\mathrm{LH}$ was added to the medium $46 \mathrm{hr}$ after the transfection, and cells were grown in the presence of $\mathrm{LH}$ for $6 \mathrm{hr}$ prior to cell harvest. Cell extracts were prepared and analyzed for CAT activity. As can be seen in Figure 9A, LH caused a dramatic stimulation of CAT activity from both VL.ovl- and VL.ov2-driven gene constructs. From radioactivity counts of the respective thinlayer chromatography spots we estimate induction of $>60$-fold. As a control, a representative LTR of another retrotransposon family, the GLN family of mouse retrotransposons (Itin and Keshet 1986b), was shown to be unresponsive to LH stimulation (Fig. 9A). These results show clearly that the inducibility of VL30 retrotransposons by gonadotropins in vivo is determined solely by LTR sequences and that inducibility is independent of the chromosomal site of residence. In addition, these experiments defined a particular subset of LTRs usable as model LH-responsive transcription units in subsequent studies.

Because the effects exerted by trophic hormones in steroidogenic cells are known to be mediated by cAMP, we wished to determine whether cAMP can induce VL30 transcription directly. To this end we have replaced LH with cAMP in the transfection experiments described in Figure 9. As can be seen, cAMP was as effective as LH in stimulating the LTR-driven CAT activity. [Other experiments have also shown that cAMP was as effective as LH in stimulating transcription of resident VL30s (data not shown).]

VL30 LTR sequences confer LH inducibility on adjacent heterologous promoters

It has been shown repeatedly that the enhancers con-
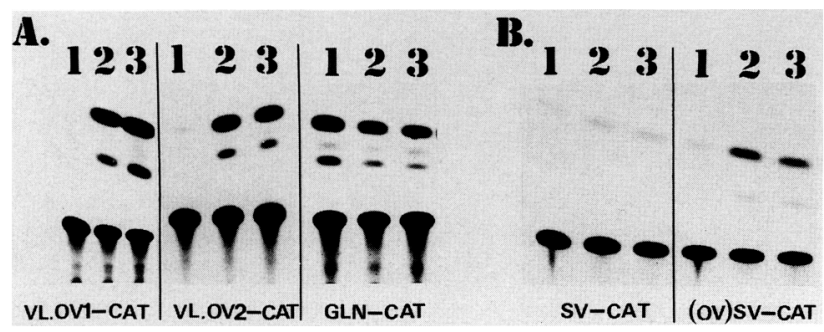

Figure 9. Transient CAT activity of VL30 LTR-CAT constructs in MLTC-1 cells depicting LH inducibility and enhancer function. Details concerning plasmid constructions and transfection protocols are provided in Materials and methods. Fortysix hours after transfection, medium was replaced with medium containing the indicated inducer, and $6 \mathrm{hr}$ later cells were harvested and processed for CAT analysis. DNAs: $(A)$ VL.ovl-CAT and VL.ov2-CAT are composed of the respective VL30 cDNAs ligated in front of a promoterless CAT gene. In the GLN-CAT control, a complete LTR unit of a GLN retrotransposon is ligated in front of the promoterless CAT. $(B)$ In SV-CAT the reporter is driven by an enhancerless SV40 early promoter (pA10-CAT derivative) (Gorman et al. 1982). In (ov)SV-CAT the cDNA of VL.ovl was inserted into the SV-CAT plasmid in an opposite orientation relative to the CAT gene. (Lanes 1) No addition; (lanes 2) LH (100 ng/ml) added to medium; (lanes 3) dibutyrl-cAMP (0.5 $\mathrm{mm}$ ) added to medium. tained in retroviral LTRs may affect the expression of nearby cellular genes. "Enhancer insertion" effects have been demonstrated with LTRs that have been inserted through both exogenous infection or through retrotransposition of cellular elements. Phenotypic consequences have been evident mostly in association with cellular transformation. Recent observations have shown, however, that additional cellular phenotypes are attributable to the enhancer action of neigboring LTRs, including hormone responsiveness of a gene that is imposed by a retrotransposon enhancer situated $>2 \mathrm{~kb}$ away from its promoter (Stavenhagen and Robins 1988).

We wished to determine whether VL30 elements have the potential to confer a LH-inducible phenotype upon adjacent genes. To this end we tested whether VL.ovl LTR confers LH inducibility on a noninducible surrogate promoter in a transient transfection assay. VL.ovl LTR DNA was inserted into a construct in which a CAT reporter gene is driven by the SV40 early promoter (but lacking the SV40 enhancer domain). DNAs were transfected into MLTC-1 cells, and transient CAT activity was determined. As can be seen in Figure 9B, the nearby presence of the LTR, in opposite orientation relative to the SV40 promoter, rendered the SV40 promoter $\mathrm{LH}$ inducible. We conclude that VL30 LTRs have the potential to impose a gonadotropin-inducible phenotype upon cellular genes located close to their sites of residence. Consistent with a CAMP-mediated pathway of LH induction, the LTR enhancer could be stimulated directly by cAMP (Fig. 9B).

\section{Discussion}

In this study we have shown that VL30 retrotransposons are specifically expressed in steroidogenic cells within all organs and tissues that specialize in synthesis of steroid hormones. Expression was mostly confined to steroid-producing cells within the ovary, the testes, the adrenal gland, and the placenta. Activation of the retrotransposons in the gonads was shown to be gonadotropin inducible both under physiologic in vivo and in vitro settings. The oscillating patterns of VL30 expression during ovarian cycles in vivo, as well as direct demonstration of gonadotropin-stimulated expression in pure cultures of steroidogenic cells in vitro, proved that the restricted pattern of expression does not merely reflect a restricted cell type-specific expression but is due to hormone responsiveness of the LTRs. In principle, a seemingly gonadotropin-inducible expression of the retrotransposon also could have been caused by a hormoneinducible cellular enhancer conferring this phenotype upon the retrotransposon (a position effect). The transfection experiments, however, in which reintroduced cloned LTR units were shown to direct the expression of a reporter gene in an LH-responsive manner clearly ruled out position effects. These experiments established that the response to the gonadotropin is an intrinsic property of the LTR.

It should be pointed out that the apparent hormone responsivness of VL30 LTRs is of different specificity 
from that of previously characterized hormone responsive LTRs, including the prototypic hormone responsive LTRs of the mouse mammary tumor virus (MMTV) (for review, see Yamamoto 1985; Beato 1989) and of murine leukemia virus (MuLV)-related proviruses (Miksicek et al. 1986); whereas the hormone response elements (HREs) of MMTV and MuLV respond to steroid hormones like glucocorticoids, androgens, and progestrins (Darbe et al. 1986; Cato et al. 1987; Glover and Darbe 1989 ) in a large number of cells carrying the respective receptors. VL30 retrotransposons are apparently stimulated by nonsteroid hormones, such as gonadotropins (and likely also ACTH), specifically in cells that possess receptors to these trophic hormones and respond by activating a complex steroidogenic pathway. Theoretical$\mathrm{ly}$, it is possible that activation of VL30 retrotransposons represents a direct response to steroid hormones operating via an autocrine mechanism within the steroid producer cell. If this possibility is correct, our observations would have suggested that all steroidogenic cells also express receptors that mediate responses to the hormones that they produce. Furthermore, the observations with VL30s would have also suggested that in cells that produce a particular steroid hormone the entire repertoire of genes that are potentially inducible by that steroid are also activated. We consider the possibility that the activation of VL30s in steroidogenic cells is mediated by steroid hormones through an autocrine mechanism as unlikely from the following considerations. First, VL30 elements are activated in vivo also in cells that produce predominantly biologically inactive steroids like cells of the embryonic adrenal. Second, VL30s are not induced in cells known to produce large amounts of glucocorticoids, like cells of the adrenal zona fasciculata (while at the same time they are induced in adjacent cells of the zona reticularis). Third, the LH inducibility of VL30s is in sharp contrast to the gonadotropin unresponsiveness of the $A k v-1$ ecotropic provirus of $\mathrm{AKR} / \mathrm{J}$ mice that is known to possess a steroid HRE and, moreover, to be simulated by steroid hormones in vitro (Celander and Haseltine 1987). Fourth, in a series of experiments that specifically address this issue, we were able to demonstrate that addition of progesterone to steroidogenic MLTC-1 cells (progesterone is the end steroid produced by these cells in culture) in the absence of LH had no stimulatory effect on VL30 expression. Furthermore, the addition of a steroid-receptor blocker (RU486), as well as the addition of aminoglutethimide, a potent inhibitor of the key steroidogenic enzyme cytochrome $\mathrm{p} 450_{\mathrm{scc}}$, resulted in effective inhibition of overall steroidogenesis and had no inhibitory effect on the LH-induced VL30 expression (R. Schiff and E. Keshet, in prep.). We conclude that activation of VL30s in steroidogenic cells is not mediated by the HRE for steroid hormones. It is reasonable to assume, therefore, that VL30 retrotransposons are directly activated by the gonadotropins.

Cells in which the retrotransposon is activated elaborate steroid hormones. Yet, the end products of the steroidogenic pathway in each of the four steroid-expressing endocrine tissues are different: Theca cells produce tes- tosterone (which is activated to estrogens in granulosa cells|, corpora lutea produce progesterone, placental cells produce progesterone, the end product of Leydig cells in vivo is testosterone, and the zona reticularis cells of the adrenal cortex produce primarily androgens. At least two major steps in the synthesis of steroids are common to all steroidogenic tissues-conversion of cholesterol to pregnenolone by the action of a mitochondrial P450 system, and conversion of pregnenolone to progesterone by the action of two microsomal enzymatic activities. It is conceivable that VL30 elements are activated by the same trans-acting factors that also activate one or more steroidogenic enzymes. This could have been a consequence of the evolution of certain LTRs in a way that the appropriate sequence domains that are both necessary and sufficient for this specific activation have been generated and maintained in the LTR. The trophic hormones in question are known to exert their biologic action via a cAMP-mediated pathway. A trophic hormoneinduced CAMP-mediated pathway is known to be activated in every steroidogenic cell type shown by this study to activate VL30. We have shown clearly that addition of cAMP to cultured steroidogenic cells resulted in dramatic activation of both resident and reintroduced retrotransposons. The potential inducibility of VL30 retrotransposons by cAMP in lymphocytes has been shown recently (Harrigan et al. 1989).

Our success in cloning gonadotropin-inducible transcription units proved to be activated in steroidogenic cells in vivo provides an excellent model for studying transcription units displaying such specificity with the aim of delineating cis-acting sequences that mediate inducibility and for studying interactions with trans-acting factors. In this regard the LTRs are an important addition to the few trophic hormone-responsive transcription units currently available as molecular clones. Interestingly, both the gonadotropin-inducible promoters (Hickey et al. 1990) and an ACTH-inducible promoter (Rice et al. 1989) contain cAMP-responsive element (CRE)-like elements. None of these elements fully matches the CRE consensus sequence. The fact that all necessary cis-acting regulatory domains are contained within a relatively short U3 segment of the retrotransposon LTR should facilitate the analysis.

Another potential utility of these inducible retrotransposons is as in situ markers of cells actively engaged in steroid biosynthesis and in studies of the tissue and organ distribution of steroidogenic cells. The current study has provided two examples for the feasibility of this approach. First, the observation that in the adrenal gland the retrotransposon is activated only in a subset of cells that is capable of producing steroids is consistent with the notion of functional zonation of cortical cells but precedes the knowledge of precise distribution of cells with respect to their different steroidogenic outputs. Second, the dynamic changes in cell populations expressing the retrotransposon during the processes of follicular maturation and placentation illustrate the utility of retrotransposons as marking those cells that are exercising their steroidogenic potential. In addition, the retrotrans- 
posons are potentially useful as developmental in situ probes marking the onset of steroidogenesis in different embryonic compartments.

No programmed role has been assigned so far to retrotransposons in animal cells. Retrotransposons may nevertheless affect cellular phenotypes in a nonprogrammed fashion in a number of ways. From their numerous sites of residence, retrotransposons may potentially affect the pattern of expression of a large number of cellular genes. It is likely that the phenomenon of cellular genes whose apparent hormone inducibility is imposed by a nearby retrotransposon (Stavenhagen and Robins 1988) is much more widespread than appreciated from incidental encounters. Our results, that at least a subset of VL30 elements are capable of imposing gonadotropin responsiveness on nearby promoters, when viewed in conjuction with the abundance of retrotransposons in the mouse genome in general, suggest that additional hormone-responsive phenotypes might be due to the hormone-dependent enhancers of nearby retrotransposons.

Finally, the ability of retrotransposons to reinsert themselves in new cellular loci adds more opportunities for enhancer insertions. It has been proposed that the frequent germ-line acquisitions of new ecotropic proviruses in certain combinations of mouse strains are mediated by activation of these infection-competent proviruses in the vicinity of the oocyte (Jenkins and Copeland 1985; Lock et al. 1988; Panthier et al. 1989). Because VL30 retrotransposons are also capable of cell-to-cell transmission-provided that they are supplemented with trans-acting retroviral proteins-it is possible that their coexpression in the gonads with other endogenous proviruses will lead to their germ-line transmission. A possible outcome of these transmissions might be the acquisition of a hormone-inducible expression by additional genes.

\section{Materials and methods}

Animals, embryos, and organs

Eight-week-old $\mathrm{C} 57 / \mathrm{Bl}$ mice were used unless indicated otherwise. Hyperstimulation of ovaries was induced by injecting, intraperitoneally (IP), 5 IU of PMSG (GC-2; Sigma), followed $44 \mathrm{hr}$ later by injection of 5 IU of hCG (G-4877; Sigma). For derivation of gonadotropin-stimulated testes, 5 IU of hCG was injected into 10 -week-old mice, and the testes were removed $5 \mathrm{hr}$ later. To obtain embryos at successive stages of development, matings were set and embryos were staged as 0.5 -day p.c. at noon of the day on which the vaginal plug was observed.

\section{Cells}

The MLTC-1 cell line was derived from a Leydig tumor (Rebois 1982), and cells were grown as described by Rebois (1982). For induction of steroidogenesis ovine LH (NIDDK-oLH-24; LH potency $=2.3 \mathrm{NIH}-\mathrm{LH}-\mathrm{Sl} \mathrm{U} / \mathrm{mg}$; FSH contamination $<0.5 \%$ by weight) a gift from the NIDDK, Bethesda, MD) was added to the medium, as indicated in the legend to Figure 7.

\section{In situ hybridization}

For in situ hybridization either uterine segments containing early postimplantation embryos $(<10.5$ days p.c.), whole embryos, or selected organs were removed and placed in $4 \%$ paraformaldehyde in phosphate-buffered saline (PBS); they were fixed overnight at $4^{\circ} \mathrm{C}$. Fixed organs were then incubated overnight in $0.5 \mathrm{M}$ sucrose in PBS prior to embedding in tissue-tek O.C.T. embedding medium (Miles Scientific) and in situ hybridization. Specimens intended for RNA extraction were removed, placed in PBS, and frozen immediately.

In situ hybridization was performed essentially as described by Hogan et al. (1986). Briefly, 10- $\mu$ m-thick frozen sections were collected on poly-L-lysine-coated glass slides, refixed in $4 \%$ paraformaldehyde, and dehydrated in graded ethanol solutions. Before hybridization, sections were pretreated sucessively with $0.2 \mathrm{~N} \mathrm{HCl}, 2 \times \mathrm{SSC}, 0.125 \mathrm{mg} / \mathrm{ml}$ of pronase, $4 \%$ paraformaldehyde, and acetic anhydride in triethanolamine buffer. Hybridization was carried out at $50^{\circ} \mathrm{C}$ overnight in $50 \%$ formamide, 0.3 $\mathrm{M} \mathrm{NaCl}$ containing $10 \%$ dextran sulfate, $1 \times$ Denhardt's solution, $1 \mathrm{mg} / \mathrm{ml}$ of carrier tRNA, $10 \mathrm{~mm}$ DTT, $5 \mathrm{~mm}$ EDTA, and $2 \times 10^{8} \mathrm{cpm} / \mathrm{ml}$ of ${ }^{35} \mathrm{~S}$-labeled riboprobe. Posthybridization washing was performed under stringent conditions that included an incubation at $50^{\circ} \mathrm{C}$ for $>14 \mathrm{hr}$ in $50 \%$ formamide $/ 0.3$ $\mathrm{M} \mathrm{NaCl}$, and a $30-\mathrm{min}$ incubation at $37^{\circ} \mathrm{C}$ in $20 \mu \mathrm{g} / \mathrm{ml}$ of RNase A. Autoradiography was performed by using Kodak NTB-2 nuclear track emulsion.

\section{Hybridization probes}

As a source of the VL30-specific probe, a 350-bp-long LTR fragment derived from the genomic clone VL5 (Itin and Keshet 1986) was used. A $X b a I-S m a I$ fragment was subcloned into the Bluescribe (pBS) vector (Stratagene) and was linearized with the appropriate restrection enzyme to allow synthesis of a ${ }^{35} \mathrm{~S}-1 \mathrm{a}$ beled RNA in the antisense and sense orientations /using T7 or T3 polymerase, respectively).

The ecotropic virus-specific probe was a 330-bp-long fragment derived from an ecotropic MuLV clone (Chattopadhyay et al. 1980) and subcloned in pBS.

\section{Blot hybridization analysis of RNA}

Total RNA was extracted from tissues homogenized in $\mathrm{LiCl}$ / urea solution. RNA was denatured in formaldehyde and electrophoresed through 1.3\% agarose/formaldehyde gel in MOPS buffer. RNAs were transferred to a nylon-based membrane (Genescreen, NEN) and hybridized with the indicated probes. Probes were labeled with $\left[{ }^{32} \mathrm{P}\right] \mathrm{dCTP}$ by using a multiprime DNA labeling system (Amersham). For standardization, filters were rehybridized with a $\beta$-actin-specific probe (Minty et al. 1981).

\section{Nuclear run-off transcription}

The nuclear run-off transcription assay was carried out as described by Greenberg and Ziff (1984). There were minor modifications made to the technique.

\section{cDNA cloning and sequencing}

Ovaries were removed from mice $5 \mathrm{hr}$ after hCG injection. Polyadenylated RNAs were purified, and double-stranded cDNAs were synthesized by using components of a cDNA synthesizing kit (Amersham, no. RPN.1256). EcoRI linkers were added, cDNAs were methylated in vitro, digested with EcoRI, ligated with $\lambda$ gt 10/EcoRI vector DNA (Stratagene), and packaged to form infectious phages using packaging extracts (Gigapack II gold, Stratagene). 
Library screening was carried out with the VL30 LTR-specific probe described above. cDNA inserts were subcloned into a pBS vector (Stratagene), and cDNAs were sequenced by the dideoxy chain-terminatation method.

\section{DNA construction}

For preparing reporter gene constructs two VL30 cDNA clones, designated VL.ovl and VL.ov2, were used. The two starting plasmids were composed of the respective VL30 sequences cloned into the EcoRI site of the pBS polylinker. Other sites of the polylinker were then utilized to insert CAT-containing fragments in a manner that juxtapositioned the reporter gene downstream of the LTR U3/R region. In constructs designed to analyze VL30 promoters (VL.ovl-CAT and VL.ov2-CAT) the inserted CAT fragment was devoid of promoter sequences, whereas in the construct designed to analyze VL30 enhancer function [(ov)SV-CAT] the inserted CAT fragment included a SV40 early promoter region from which enhancer sequences had been deleted [pA10-CAT (Gorman et al. 1982)]. In the latter case, the CAT-containing fragment was inserted in opposite orientation relative to the VL30 sequences. The control SVCAT plasmid contains no VL30 sequences but is otherwise identical to (ov)SV-CAT. GLN-CAT contains a complete LTR unit of a GLN retrotransposon (Itin and Keshet 1986b) as a promoter/enhancer unit.

\section{Transfections}

MLTC- 1 cells were plated at a density of $7 \times 10^{5}$ cells $/ 60-\mathrm{mm}$ plate $48 \mathrm{hr}$ prior to transfection. For each transfected plate $10 \mu \mathrm{g}$ of DNA was diluted with $50 \mu \mathrm{g}$ of Lipofectin reagent (BRL no. 8292SA), and complexes were added to cells in a serum-free medium under conditions recommended by the manufacturer. An equal volume of RPMI medium containing $20 \%$ fetal calf serum was added $6 \mathrm{hr}$ later. Cells were harvested $50 \mathrm{hr}$ posttransfection. The indicated inducing agents were added $6 \mathrm{hr}$ prior to cell harvest. Preliminary studies have indicated that transfection of MLTC-1 cells by this procedure is more efficient than other commonly used transfection procedures.

\section{Acknowledgments}

This work was supported by grants from the Ministry of Science and Technology, Israel, and the Commission of the European Community, and from the Israel-U.S.A. Binational Science Foundation.

The publication costs of this article were defrayed in part by payment of page charges. This article must therefore be hereby marked "advertisement" in accordance with 18 USC section 1734 solely to indicate this fact.

\section{References}

Anderson, G.R., D.L. Stoler, and L.A. Scarcello. 1989. Retrotransposon-like VL30 elements are efficiently induced in anoxic rat fibroblasts. J. Mol. Biol. 205: 765-769.

Beato, M. 1989. Gene regulation by steroid hormones. Cell 56: 335-344.

Besmer, P., U. Olshevski, D. Baltimore, D. Dolberg, and H. Fan. 1979. Virus-like 30S RNA in mouse cells. J. Virol. 29: 11681176.

Boeke, J.M., D.J. Garfinkel, C.A. Styles, and G.R. Fink. 1985. Ty elements transpose through an RNA intermediate. Cell 40: 491-500.
Cato, A.B.C., D. Handerson, and H. Ponta. 1987. The hormone response element of the mouse mammary tumor virus DNA mediates the progestin and androgen induction of transcription in the proviral long terminal repeat. EMBO J. 6: 363368.

Celander, D. and W.A. Haseltine. 1987. Glucocorticoid regulation of murine leukemia virus transcription elements is specified by determinants within the viral enhancer region. J. Virol. 61: 269-275.

Chattopadhyay, S.K., M.R. Lander, E. Rands, and D.R. Lowy. 1980. Structure of endogenous murine leukemia virus DNA in mouse genomes. Proc. Natl. Acad. Sci. 77: 5774-5778.

Darbe, P., M. Page, and R.J.B. King. 1986. Androgen regulation by the long terminal repeat of MMTV. Mol. Cell. Biol. 6: 2847-2854.

Foster, D.N., L.J. Schmidt, C.P. Hodgson, H.L. Moses, and M.J. Getz. 1982. Polyadenylated RNA complementary to mouse retrovirus-like multigene family is rapidly and specifically induced by epidermal growth factor stimulation of quiescent cells. Proc. Natl. Acad. Sci. 79: 7317-7321.

Glover, J.F. and P.D. Darbe. 1989. Multihormone regulation of MMTV-LTR in transfected T-47-D human breast cancer cells. I. Steroid. Biochem. 32: 357-363.

Gorman, C.M., L.F. Moffat and B.H. Howard. 1982. Recombinant genomes which express chloramphenicol acetyltransferase in mammalian cells. Mol. Cell. Biol. 2: 1044-1051.

Greenberg, M.E. and E.B. Ziff. 1984. Stimulation of 3T3 cells induces transcription of the c-fos proto-oncogene. Nature 311: 433-437.

Hsieh, L.L., W.-L. Hsiao, C. Peranio, R.R. Maronpot, and I.B. Weinstein. 1987. Expression of retroviral sequences and oncogenes in rat liver tumors induced by diethylnitrosamine. Cancer Res. 47: 3421-3424.

Howk, R.S., D.H. Troxler, D. Lowy, P.H. Duesberg, and E.M. Scolnick. 1978. Identification of a 30S RNA with properties of a defective type $\mathrm{C}$ virus in murine cells. $J$. Virol. 25: 115123.

Harrigan, M.T., G. Baughman, F. Campbell, and S. Bourgeois. 1989. Isolation and characterization of glucocorticoid- and cyclic AMP- induced genes in T lymphocytes. Mol. Cell. Biol. 9: 3438-3446.

Hickey, G.J., J.S. Krasnow, W.G. Beattie, and J.S. Richards. 1990. Aromatase cytochrome $\mathrm{p} 450$ in ovarian granulosa cells before and after leutinization: Adenosine $3^{\prime} 5^{\prime}$ monophosphate dependent and independent regulation: Cloning and sequencing of aromatase cDNA and 5' genomic DNA. Mol. Endocrinol. 4: 3-11.

Hogan, B., F. Constantini, and E. Lacy. 1986. Manipulating the mouse embryo. Cold Spring Harbor Laboratory, Cold Spring Harbor, New York.

Itin, A. and E. Keshet. 1986a. Diverse long terminal repeats are associated with murine retrovirus-like elements. Mol. Cell. Biol. 6: 1276-1282.

- 1986b. A novel retroviruslike family in mouse DNA. J. Virol. 59: 301-307.

Itin, A., G. Rotman, and E. Keshet. 1983. Apparent recombinants between virus-like and murine leukemia virus related sequences in mouse DNA. J. Virol. 47: 178-184.

Jenkins, N.A. and N.G. Copeland. 1985. High frequency germline acquisition of ecotropic MuLV proviruses in SWR/J-RF/J mice. Cell 43: 811-819.

Jenkins, N.A., N.G. Copeland, B.A. Taylor, and B.K. Lee. 1982. Organization, distribution, and stability of endogenous ecotropic murine leukemia virus DNA sequences in chromosomes of Mus musculus. I. Virol. 43: 26-36.

Keshet, E., R. Schiff, and A. Itin. 1990. Murine retrotransposons: 
A cellular reservoir of long terminal repeat (LTR) elements with diverse transcriptional specificities. Adv. Cancer Res. 56: .

Keshet, E., Y. Shaul, J. Kaminchik, and H. Aviv. 1980. Heterogeneity of retrovirus-like genes and their organization within the mouse genome. Cell 20: 431-439.

Lock, L.F., E. Keshet, D.J. Gilbert, N.A. Jenkins, and N.G. Copeland. 1988. Studies on the mechanism of spontaneous germline ecotropic provirus acquisition in mice. EMBO $\mathrm{J}$. 7: 4169-4177.

Miksicek, R., A. Heber, W. Schmid, U. Danesch, G. Posseckert, M. Beato, and G. Schutz. 1986. Glucocorticoid responsiveness of the transcriptional enhancer of Moloney murine sarcoma virus. Cell 46: 283-290.

Minty, J.A., M. Caravatti, B. Robert, A. Cohen, P. Dubas, A. Weydert, F. Gros, and M.E. Buckingham. 1981. Mouse actin messenger RNAs. J. Biol. Chem. 256: 1008-1014.

Oonk, R.B., J.S. Krasnow, W.G. Beattie, and J.S. Richards. 1989. Cyclic AMP-dependent and independent regulation of cholesterol side chain cleavage cytochrome p-450 (p-450 rat ovarian granulosa cells and corpora lutea. J. Biol. Chem. 264: 21934-21942.

Orly, J. 1989. Orchestrated expression of steroidogenic sidechain cleavage cytochrome $\mathrm{p}-450$ during follicular development in the rat ovary. J. Reprod. Fertil. 37(suppl.): 155-162.

Panthier, J.J. and H. Condamine. 1987. Expression of ecotropic MuLV in ovaries of SWR/J-RF/J hybrid mice. Ann. Inst. Pasteur Virol. 138: 409-422.

Panthier, J.J., P. Gounon, H. Condamine, and F. Gros. 1989. Pattern of expression of ecotropic murine leukemia virus in gonads of inoculated SWR/J mice. J. Virol. 63: 2134-2142.

Rebois, R.V. 1982. Establishment of gonadotropin-responsive murine Leydig tumor cell line. I. Cell. Biol. 94: 70-76.

Rice, D.A., L.D. Aitken, G.R. Vandenbark, A.R. Mouw, A. Franklin, B.P. Schimmer, and K.L. Parker. 1989. A cAMP-responsive element regulates expression of the mouse steroid 11ß-hydroxylase gene. J. Biol. Chem. 264: 14011-14015.

Richards, J.S. 1980. Maturation of ovarian follicles: Actions and interactions of pituitary and ovarian hormones on follicular cell differentiation. Physiol. Rev. 60: 51-89.

Simpson, E.R. and M.R. Waterman. 1988. Regulation of the synthesis of steroidogenic enzymes in the adrenal cortical cells by ACTH. Annu. Rev. Physiol. 50: 427-440.

Singh, K., S. Saragosti, and M. Botchan. 1985. Isolation of cellular genes differentially expressed in mouse NIH 3T3 cells and a simian virus 40-transformed derivative: Growth specific expression of VL30 genes. Mol. Cell. Biol. 5: 25902598.

Soares, M.J., J.A. Julian, and S.R. Glasser. 1985. Trophoblast giant cell release of placental lactogens: Temporal and regional characteristics. Dev. Biol. 107: 520-526.

Stavenhagen, J.B. and D.M. Robins. 1988. An ancient provinus has imposed androgen regulation on the adjacent mouse sexlimited protein gene. Cell 55: 247-254.

Yamamoto, K.R. 1985. Steroid receptor regulated transcription of specific genes and gene networks. Annu. Rev. Genet. 19: 209-215. 


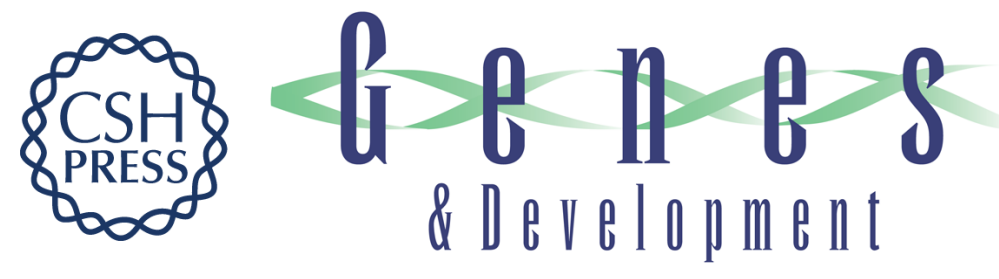

\section{Transcriptional activation of mouse retrotransposons in vivo: specific expression in steroidogenic cells in response to trophic hormones.}

R Schiff, A Itin and E Keshet

Genes Dev. 1991, 5:

Access the most recent version at doi:10.1101/gad.5.4.521

References This article cites 37 articles, 19 of which can be accessed free at: http://genesdev.cshlp.org/content/5/4/521.full.html\#ref-list-1

License

Email Alerting Receive free email alerts when new articles cite this article - sign up in the box at the top Service right corner of the article or click here.

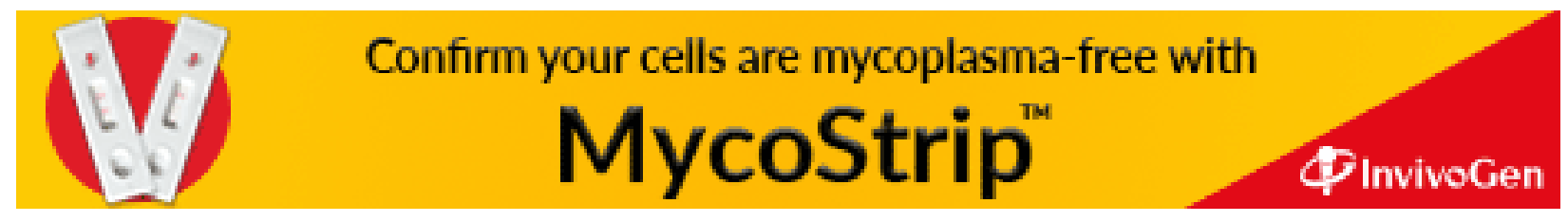

